\title{
FINE STRUCTURE OF THE BOVINE OOCYTE FROM THE MATURE GRAAFIAN FOLLICLE
}

\author{
W. NESBIT FLEMING AND R. G. SAACKE \\ Department of Dairy Science, Virginia Polytechnic Institute and State University, \\ Blacksburg, Virginia 24061, U.S.A.
}

(Received 13th April 1971, accepted 26th Fuly 1971)

\begin{abstract}
Summary. Nineteen oocytes aspirated from mature Graafian follicles of normally cycling cows in standing oestrus were studied. Oocytes were fixed in phosphate-buffered glutaraldehyde followed by exposure to osmium tetroxide and embedding in Epon 812. Oocytes could be classified into four types based on characteristics of nuclear components and distribution of organelles and inclusions. These types represented late maturational changes occurring shortly before ovulation. The close association of granulosa-cell processes in Type-I maturing follicular oocytes showed progressive signs of deterioration in other oocyte types. In all oocytes, numerous mitochondria with 'hood-like' appendages were observed. Single cisternae of endoplasmic reticulum were closely associated with the surface of mitochondria and the cavity formed by the 'hood-like' appendage. Endoplasmic reticulum was also associated with lipid droplets and was observed to be continuous with the outer leaflet of the nuclear envelope. Infrequently, a more classical granular endoplasmic reticulum was apparent. The cytoplasmic matrix was moderately dense and contained scattered ribosomes and polysomes. Abundant vesicles containing small PAS-positive granules were evenly distributed throughout the ooplasm. Golgi dictyosomes, annulate lamellae, cortical granules and two types of nucleoli characterized these oocytes.
\end{abstract}

\section{INTRODUCTION}

Attempts to harvest, preserve and transfer bovine ova successfully as well as understand the sources of reproductive failure in this species have been numerous. One aspect which appears critical to such studies but has not been closely examined is the anatomy of the normal mature bovine ovum.

It has been well established for most species, including the bovine, that the fertilization rate is generally above $90 \%$ (Asdell \& Mixner, 1957). It is also clear, from studies with pigs and rabbits, that fertilization of aged ova results in decreased ability of the zygote to sustain embryogenesis (Austin, 1967; Hunter, 1967), and that in repeat-breeding cattle, embryonic mortality is greatly increased (Tanabe \& Casida, 1949). To provide a structural basis for the evaluation of bovine ova with respect to their potential for fertilization or 
maintenance of embryogenesis, the characteristics of the ovum in final stages of maturation within the ovary must first be described.

The objective of this study was to establish the structural characteristics of the bovine oocyte recovered from the mature Graafian follicle at the time of behavioural oestrus. This oocyte, which is destined for ovulation approximately $12 \mathrm{hr}$ after cessation of oestrus, is easily identified since it is in the largest follicle on the ovaries during oestrus. Nearly all other tertiary follicles on the bovine ovary have been considered atretic, and contain oocytes in various stages of deterioration (Marion, Gier \& Choudary, 1968).

\section{PROCEDURE}

Nineteen oocytes from mature Graafian follicles were obtained from cows in the University dairy herd. Only cows which had displayed two normal oestrous cycles and had normal reproductive histories were used in the study. The cows were observed morning and afternoon for behavioural oestrus (standing while mounted by other females). Within $4 \mathrm{hr}$ of a cow first having been observed to be in oestrus, a bilateral ovariectomy was performed by laparotomy using a spaying ecrasure. The largest follicle was considered to be the mature Graafian follicle. The oocyte was quickly recovered from the follicle by aspiration using a 3- or 5-ml hypodermic syringe fitted with a 22-gauge needle. The manipulation of ova in preparation for light and electron microscopy conformed with the technique described by Senger \& Saacke (1970a). Fixation was carried out at 0 to $3^{\circ} \mathrm{C}$ in $3 \%$ glutaraldehyde buffered to $\mathrm{pH} 7.2$ to 7.4 with a $0.1 \mathrm{M}$-phosphate solution (Luce, 1966). The time from ovariectomy to the fixation of the oocytes was $11.7 \pm 5.9 \mathrm{~min}$.

After fixation for $1 \mathrm{hr}$, the oocytes were transferred to the $0.1 \mathrm{M}$-phosphate buffer and held at room temperature overnight. Oocytes were postfixed in $0.1 \mathrm{M}$-phosphate-buffered $1 \%$ osmium tetroxide followed by dehydration in a graded enthanol series and embedding in Epon 812.

Thin sections were cut with a glass or diamond knife on a Porter-Blum MT-2 ultramicrotome, stained with uranyl acetate and lead citrate according to Venable \& Coggeshall (1965), and examined with an RCA-EMU-3H electron microscope. Adjacent thick sections $(0.5 \mu \mathrm{m})$ were mounted on glass slides and stained with Azure II (Jeon, 1965). Thick serial sections were examined with the light microscope to determine the general distribution of organelles and inclusions and to relate structural details observed with the light microscope to those resolved with the electron microscope.

Localization of lipid in the oocytes was carried out by exposing $0.5 \mu \mathrm{m}$-Epon sections to a $0.5 \%$ Oil Red O stain in propylene glycol. Azure II was used as a counter-stain. Thick Epon sections were also exposed to a periodic acidSchiff staining procedure modified from Gurr (1953) for localization of carbohydrate material. Sections were placed into the $\mathbf{B}$ solution of periodic acid for $15 \mathrm{~min}$, washed in $70 \%$ alcohol then placed into a reducing rinse. Following another alcohol rinse, the sections were exposed to Schiff's reagent for $30 \mathrm{~min}$ then placed in sulphite wash and rinsed in distilled water. The sections were counter-stained with Fast Green. 


\section{RESULTS}

Although all oocytes were recovered from the mature Graafian follicle during oestrus, they could be classified into four types based upon organelle distribution, formation of the perivitelline space and structural configuration of nuclear components. The types are presented in chronological order consistent with our current understanding of late preovulatory maturation (Pl. 1, Figs. 1 to 4). The first type was characterized by a peripheral distribution of mitochondria and lipid inclusions with an eccentrically located nucleus (Pl. 1, Fig. 1) having an intact nuclear envelope (PI. 4, Fig. 12). In this type of oocyte, a perivitelline space was not apparent. The second type was characterized by a general distribution of mitochondria and lipid droplets (Pl. 1, Fig. 2A). The perivitelline space was apparent and the nucleus was unchanged relative to Type I (Pl. 1, Fig. 2B). In the third type, the nuclear envelope was no longer apparent and the chromosomes were very condensed (Pl. 1, Fig. 3). The fourth type was characterized by the presence of the first polar body in the perivitelline space (Pl. 1, Fig. 4). Mitochondria and lipid droplet distribution in Types III and IV remained general. Ten of the nineteen oocytes studied were of Type I while the remaining nine oocytes were distributed equally among the other three types, suggesting that the observed alterations are of short duration and probably occur in late oestrus. The number of granulosa cells adhering to the zona of a given oocyte ranged from very abundant to none and could not be related to the type of oocyte. Many granulosa cells were undoubtedly removed in the process of aspirating the oocyte from the follicle.

Ultrastructurally, the zona pellucida was composed of a moderately staining homogeneous material (Pl. 2, Fig. 5). Granulosa-cell processes and microvilli arising from the oocyte were observed within the zona pellucida. In thick sections stained with Azure II and examined with the light microscope, the perivitelline space appeared as a lighter staining band between the zona pellucida and ooplasm (Pl. 1, Figs. 2 to 4). Ultrastructurally, this band appeared as a flocculent layer easily differentiated from the zona pellucida (Pl. 2, Fig. 6). There was considerable variation among oocytes relative to the width of the perivitelline space.

In Type I oocytes, intimate points of association were observed between the granulosa-cell processes and the oolemma (Pl. 3, Figs. 7A, 7B). While these points could not be classified as desmosomes, they do resemble a portion of the junctional complex commonly referred to as the zonula adherens (Fawcett, 1966b). Surface contact between the cell membrane of the processes and oocyte was enhanced by a modification of the oolemma in the form of extended microvilli. Often, the microvilli extended over the processes where the latter contacted the oolemma (Pl. 3, Figs. 7A, 8) and in some instances, the processes appeared in the peripheral ooplasm still enveloped by the oolemma ( $\mathrm{Pl}$. 3, Fig. 7B). Cytoplasmic continuity between the oocyte and granulosa cell was never observed, although passage of material from one cell to another was indicated by pinocytotic vesicles along the oolemma in the vicinity of granulosacell processes (Pl. 3, Figs. 7A, 7B, 8). Many granulosa-cell processes at the junction of the zona pellucida and oolemma appeared to be degenerating based 
upon their granular appearance and lack of a distinct plasmalemma (PI. 3, Figs. 7A, 8).

In oocytes exhibiting a perivitelline space, granulosa-cell processes were not observed within the peripheral ooplasm or at the oolemma. Those processes which had previously been at the surface of the oocyte were absent from this region but their remnants could be found near the interface of the perivitelline space and zona pellucida (Pl. 2, Fig. 6 and Pl. 3, Fig. 9). Thus, the relationship of the granulosa-cell processes to the oocyte was related to the existence of a perivitelline space.

Aggregates of membrane-bound vesicles containing variable quantities of densely staining material were observed in the peripheral ooplasm of all oocytes (Pl. 4, Fig. 10). These structures conform to and undoubtedly are the previously reported cortical granules. Variation in content of densely staining material among the granules could not be associated with maturational changes.

Using the accepted terminology set forth by Mollenhauer \& Morré (1966), the oocyte Golgi complex consisted of numerous dictyosomes distributed throughout the peripheral ooplasm. The dictyosomes were quite classical, being composed of stacks of cisternae with many closely associated vesicles (Pl. 3, Fig. 7A). In light micrographs, the dictyosomes were observed as a slightly darker stained region in relation to the ooplasmic matrix (Pl. 4, Fig. 11). The most abundant form of endoplasmic reticulum appeared as smooth single cisternae closely associated with the outer surfaces of mitochondria (Pl. 5, Figs. 14 and 16, and Pl. 7, Fig. 23) and cytoplasmic inclusions (Pl. 7, Fig. 23). Infrequently, the endoplasmic reticulum was observed as stacks of cisternae with sparse numbers of ribosomes associated with the outer surface of the cisternae, resembling a rather loose form of classical rough endoplasmic reticulum (P1. 4, Fig. 13).

In all oocytes observed, a large proportion of the mitochondrial population were unusual in appearance. These mitochondria were first described by Senger \& Saacke (1970b). They possessed a 'hood-like' process or appendage arising from their surface (Pl. 5, Figs. 14, 15 and 17). The mitochondrial hood formed an extramitochondrial cavity that often appeared intramitochondrial when the plane of section passed through the hood, but not through the point of continuity between the mitochondrial cavity and the ooplasm (Pl. 5, Fig. 16). Sagittal sections through the hooded mitochondria revealed that pockets of endoplasmic reticulum entered the cavity from the cytoplasm and were closely apposed to the wall of the mitochondrial cavity (Pl. 5, Figs. 15, 17). On occasion, ribosomes were observed on the endoplasmic reticulum within the cavity formed by the mitochondrial hood (Pl. 5, Fig. 14). The endoplasmic reticulum, although loose in nature, appeared to interconnect many mitochondria and cytoplasmic inclusions (Pl. 5, Figs. 16, 17).

The prophase nucleus was observed in thirteen of the nineteen oocytes recovered. In Type I and II oocytes, the nucleus was eccentrically located and surrounded by an undulating nuclear envelope (Pl. 1, Fig. 1, 2B and Pl. 6, Fig. 19). The nuclear envelope was composed of two closely associated membranes with the inner membrane appearing slightly more electron dense than the outer (Pl. 4, Fig. 12 and Pl. 5, Fig. 18). At random points along the nuclear 
envelope, the two membranes appeared to fuse (Pl. 4, Fig. 12) and form what are classically referred to as 'nuclear pores'. Although the term 'pores' infers continuity between the ooplasm and nucleoplasm, such was not observed. However, in restricted regions of several oocytes, the outer leaflet of the nuclear envelope appeared to be continuous with the endoplasmic reticulum (Pl. 5, Fig. 18). In five oocytes (two Type III and three Type IV), the nuclear envelope was not apparent and in one oocyte (Type III), dissolution of the nuclear envelope was evident.

In Type I and II oocytes, the nucleoplasm was moderately electron dense and at times very similar to the ooplasm (Pl. 5, Fig. 18). Densely staining particles, resembling ribosomes, were often distributed uniformly throughout the nucleoplasm (Pl. 6, Fig. 19). The concentration of these particles varied among oocytes but without relation to the apparent stage of maturation.

The most prominent structure in the bovine oocyte nucleus was the nucleolus. There were two general types of nucleoli observed. One type, found in only two oocytes, was characterized by a well-developed nucleolonema having rather distended interstices (Pl. 6, Fig. 20). The other type, which was more commonly observed, was composed of an electron-dense ovoid structure partially encapsulated by a less electron-dense cover (Pl. 6, Fig. 21). This nucleolus was generally located near the nuclear envelope. Chromatin material was occasionally observed to be associated with both types of nucleoli. Serial sections through nuclei revealed that one nucleolus was present in each nucleus. An exception was the occurrence of two dense ovoid nucleoli in close proximity to one another in one oocyte. Following dissolution of the nuclear envelope, nucleoli were not found.

Annulate lamellae were observed in three oocytes, often in close association with aggregates of mitochondria. The annulate lamellae appeared as stacks of cisternae with constrictions at intervals along the membranes quite similar to those of the nuclear envelope (Pl. 7, Fig. 24).

All oocytes possessed globular inclusions which demonstrated metachromasia following staining with Azure II. When thick $(0.5 \mu \mathrm{m})$ sections were exposed to Oil Red $\mathrm{O}$ for $30 \mathrm{~min}$, the droplets which demonstrated metachromasia were Oil Red O-positive, confirming their lipid composition. The lipid droplets were peripherally located in Type I oocytes, but randomly distributed in more mature oocytes. The size of the droplets varied within as well as among oocytes. Mitochondria, which appeared as densely staining granules in the light microscope, were aggregated about the lipid droplets in abundant quantities (Pl. 7, Fig. 22). The cisternae of the endoplasmic reticulum were very closely associated with the lipid droplets and surrounding mitochondria (Pl. 7, Fig. 23) in all oocytes examined. During the shift in organelle distribution (peripheral to general), the relationship between mitochondria, lipid and endoplasmic reticulum was maintained.

Abundant vesicles containing densely staining granules were found in all oocytes recovered (P1. 7, Fig. 24). In the light microscope, these inclusions appeared as small vacuoles containing a flocculent material (Pl. 7, Fig. 22). This material was the only PAS-positive component of the cell which could be 


\section{EXPLANATION OF PLATES 1 TO 4}

\section{PLATE 1}

Figs. 1 to 4. Light micrographs of sectioned bovine oocytes stained with Azure II showing characteristics of the four types of oocytes recovered from mature Graafian follicles. Fig. 1. Type-I oocyte demonstrating a peripheral distribution of mitochondria, lipid droplets, and an eccentrically located nucleus $(\mathrm{N}) . \times 1595$.

FIG. 2A. Type-III oocyte, showing a random distribution of mitochondria, inclusions, and a perivitelline space (arrow) $\times 1595$.

FIG. 2B. The nucleus (N) of a Type-II oocyte revealing characteristics similar to those of Type-I oocytes. $\times 2580$.

FIG. 3. Type-III oocytes showing the condensed chromosomes associated with dissolution of the nuclear envelope. The perivitelline space (arrow) is quite obvious at this stage. $\times 1595$.

FIG. 4. Type-IV oocytes characterized by appearance of the first polar body (PB) in the perivitelline space (arrow). $\times 1595$.

\section{PLATE 2}

FIG. 5. Electron micrograph showing the zona pellucida $(Z)$ very close to the oocyte surface in a Type-I oocyte. Remnants of granulosa-cell (GC) processes are observed in the zona pellucida. $\times 5900$.

Frg. 6. The perivitelline space (PS), which appears as a flocculent layer between the zona pellucida $(Z)$ and the oocyte, characterizes Type-II, -III, and -IV oocytes. A granulosacell process (GP) is observed at the edge of the zona pellucida within the perivitelline space. $\times 1500$.

\section{PLATE 3}

Figs. 7A and B. The close relationship of the granulosa-cell process and the oolemma before formation of the perivitelline space is illustrated in these micrographs. Cross sections of the granulosa-cell processes are found surrounded by ooplasm just beneath the oolemma before formation of the perivitelline space. Pinocytotic vesicles $(P)$ are apparent in the oolemma adjacent to granulosa-cell processes and at the base of 'fingerlike' microvilli. Structures similar to the zonula adherens (ZA) of the junctional complex apparently aid in maintaining the association between the oocyte and granulosa cell. Microvilli also help to maintain this association by extending over the process. Golgi dictyosomes $(G)$ are found aggregated just beneath the oolemma throughout the peripheral cytoplasm. Ultrastructurally, the Golgi dictyosome is composed of stacks of cisternae surrounded by many vesicles. $\times 13,400$ and $\times 24,400$.

Frc. 8. Before perivitelline space formation, the granulosa-cell processes associated with the surface of the ooplasm appear to be degenerating due to their very granular appearance and lack of a plasmalemma. Pinocytotic vesicles (P) are apparent adjacent to the processes. Numerous microvilli are closely associated with the processes. $\times 14,700$. FIG. 9. After formation of the perivitelline space (PS), the degenerating granulosa-cell processes are found at the edge of the zona pellucida $(Z)$ within the perivitelline space. $\times 11,840$.

\section{PLATE 4}

FIG. 10. Cortical granules (CG) showing variation in content of densely staining material. The granules were found in aggregates throughout the peripheral ooplasm just beneath the oolemma. $\times 12,900$.

Fig. 11. A light micrograph of an Azure II-stained section showing a dictyosome of the Golgi complex $(G)$. This region appears as a moderately dense stained area close to the oolemma. × 3705 .

Fig. 12. The nuclear envelope is composed of two membranes closely associated with one another. Fusion of the two membranes at random points along the envelope form what is classically referred to as 'nuclear pores' (arrows). $\times 52,500$.

FIG. 13. Endoplasmic reticulum (ER) is occasionally observed in loose stacks of cisternae studded with ribosomes and closely associated with the surface of mitochondria. Numerous ribosomes and polysomes are apparent throughout the moderately electron-dense ooplasmic matrix. $\times 11,350$. 

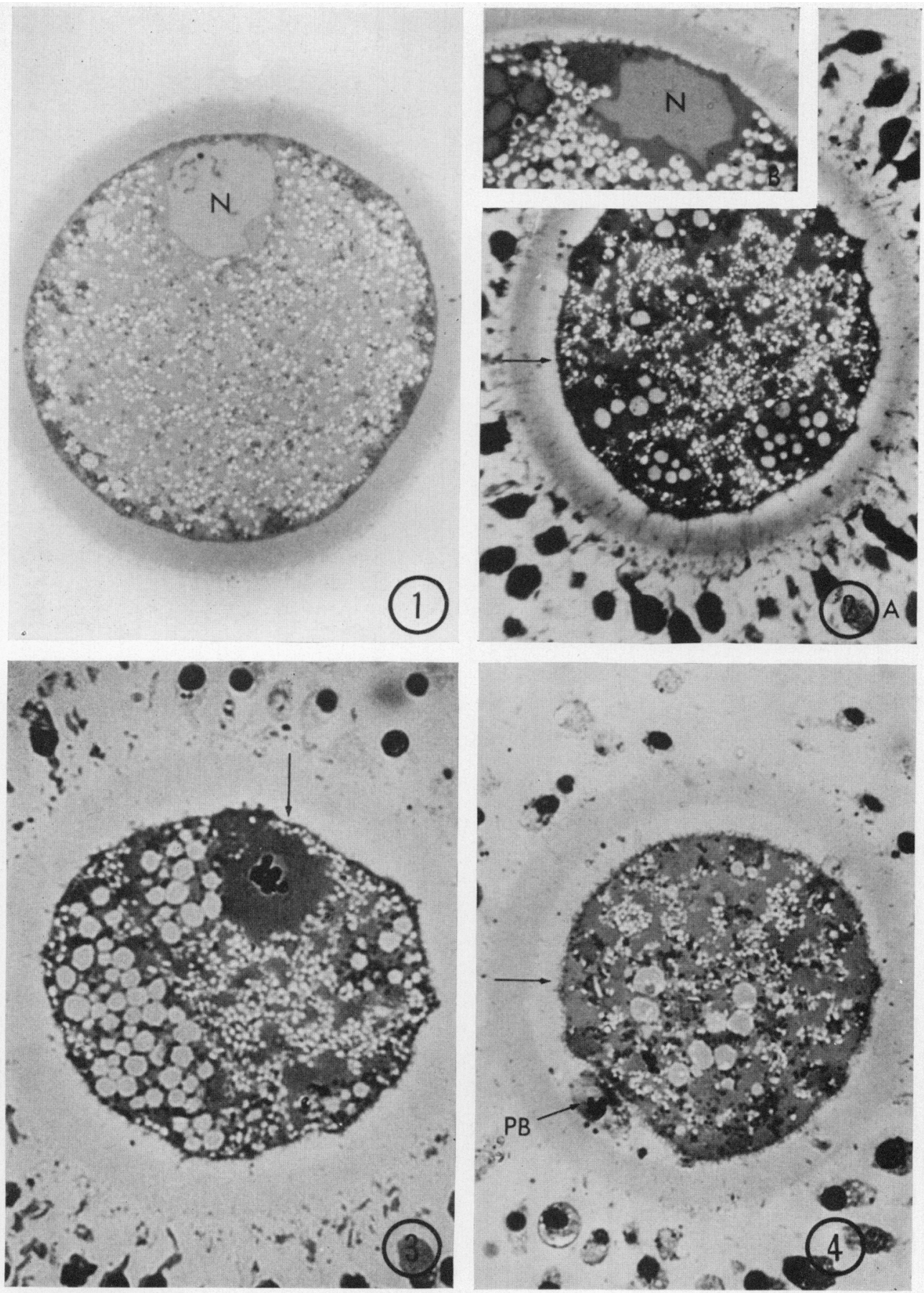


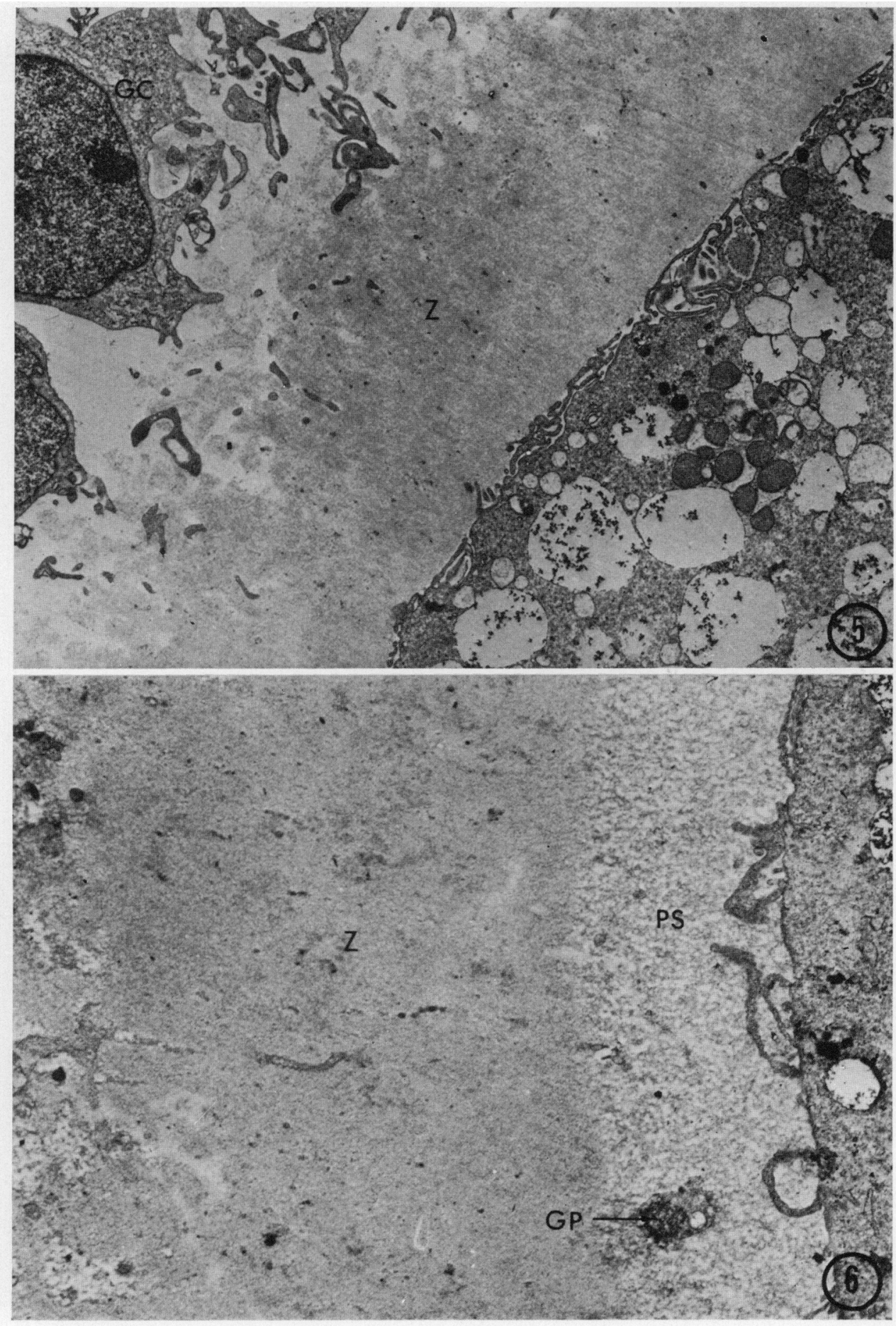


PIATE 3
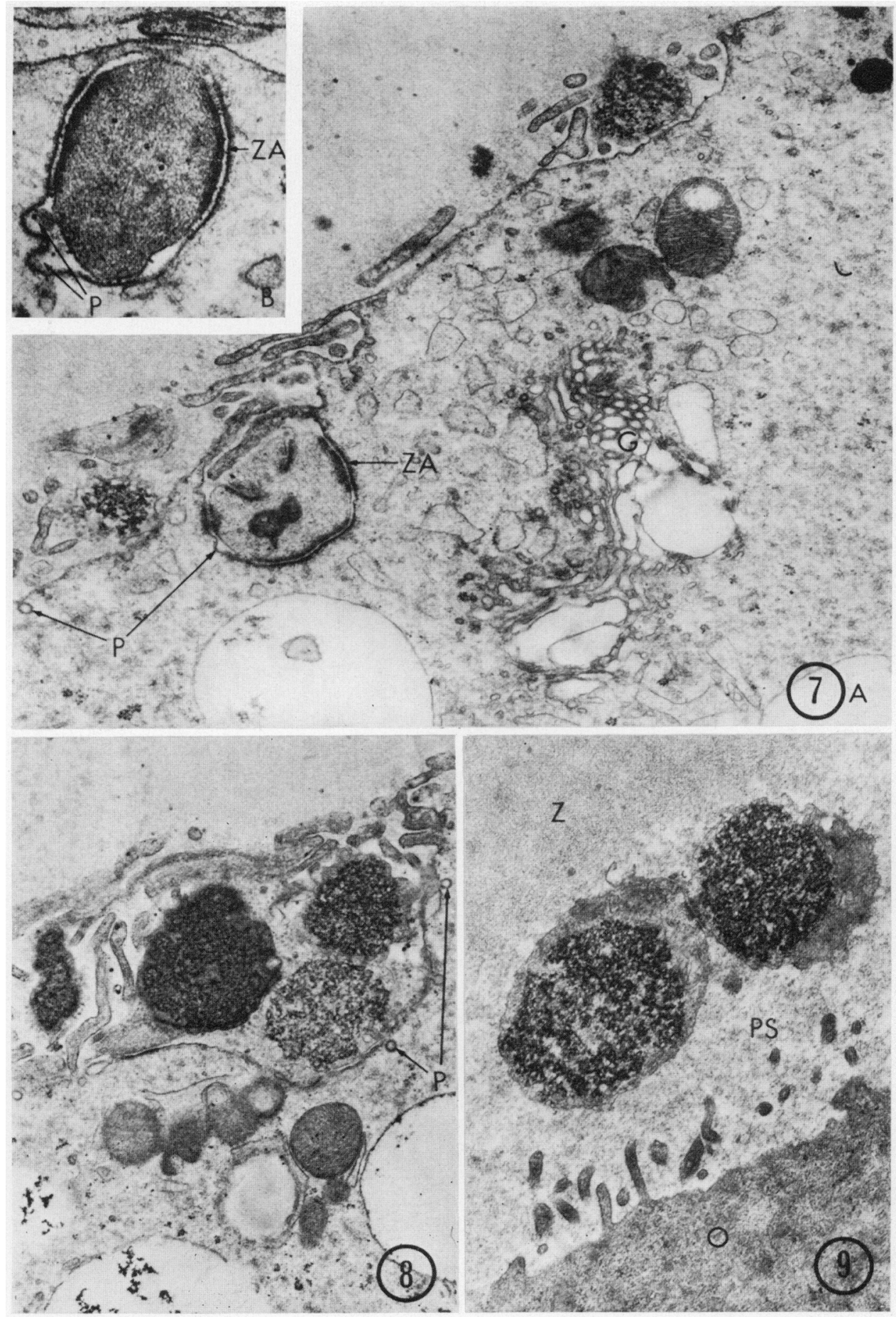


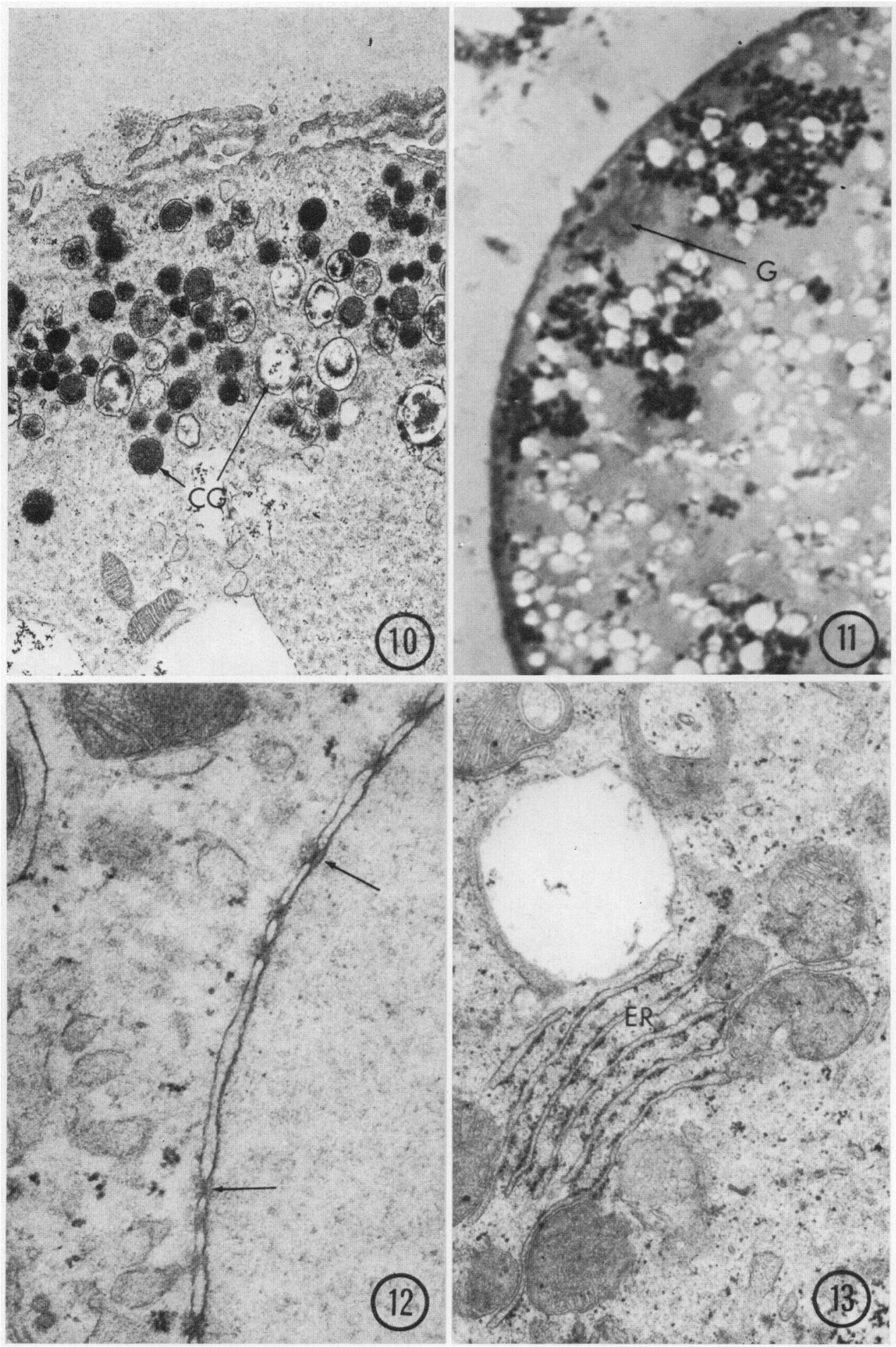


PIIIE
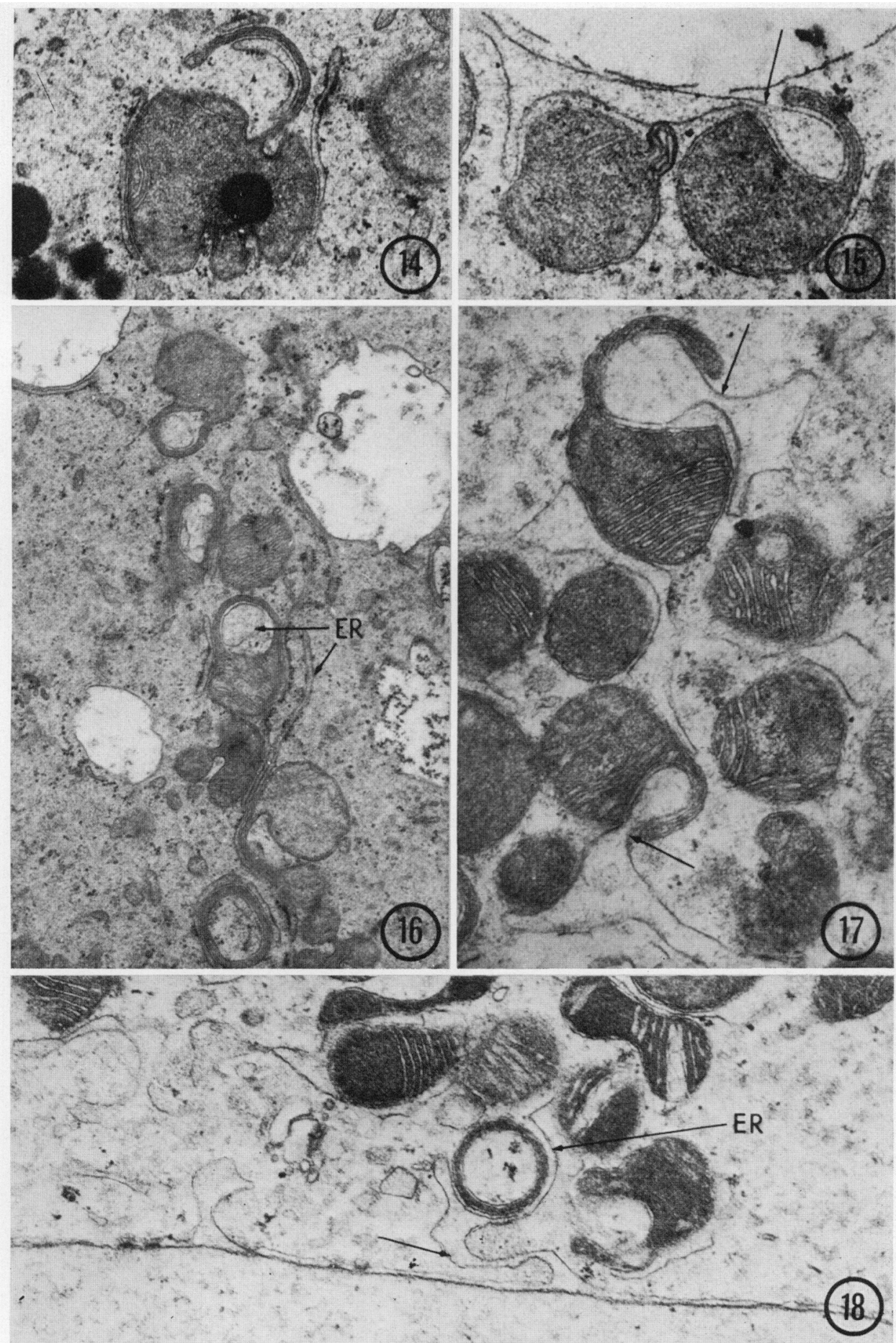
PLATE 6

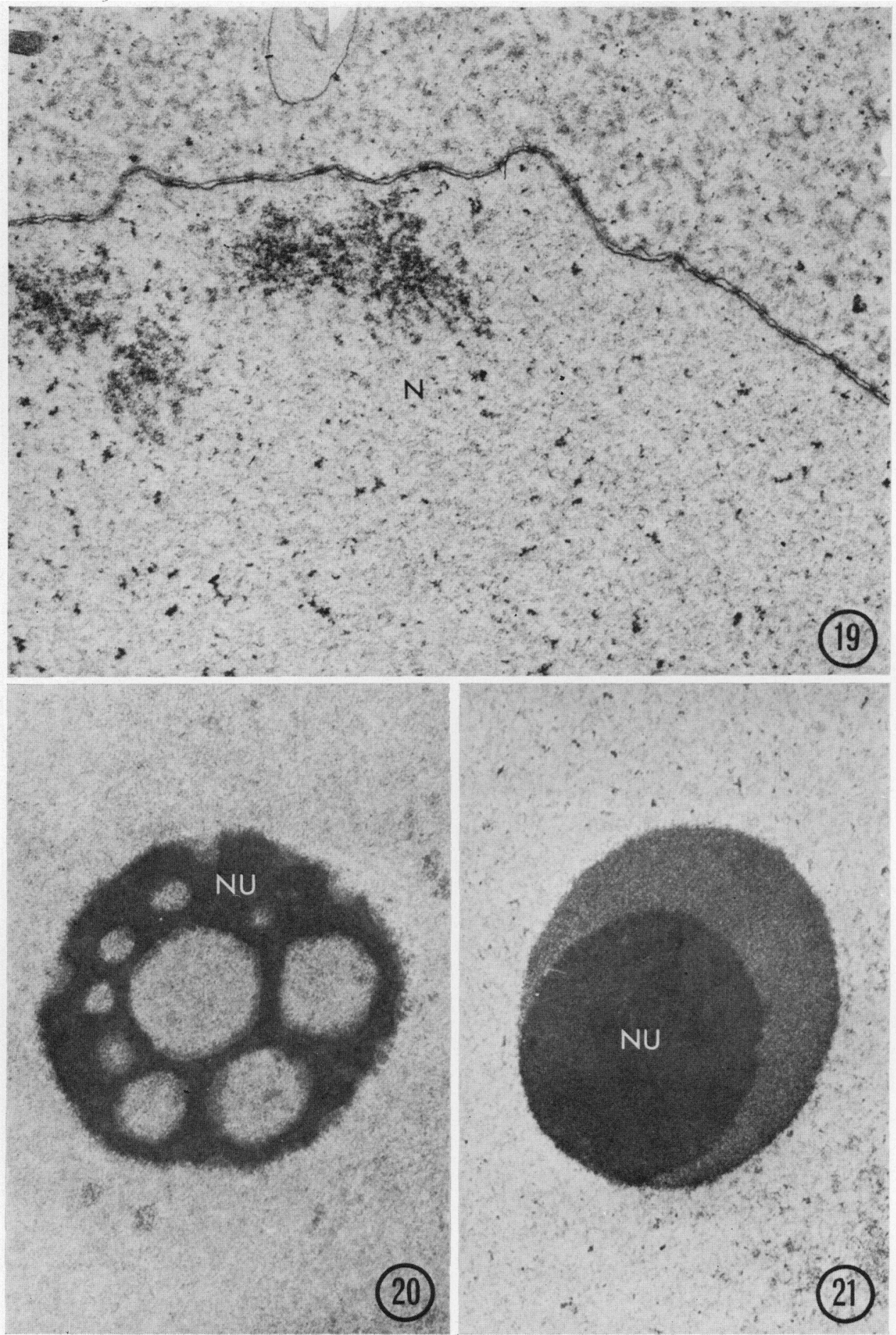



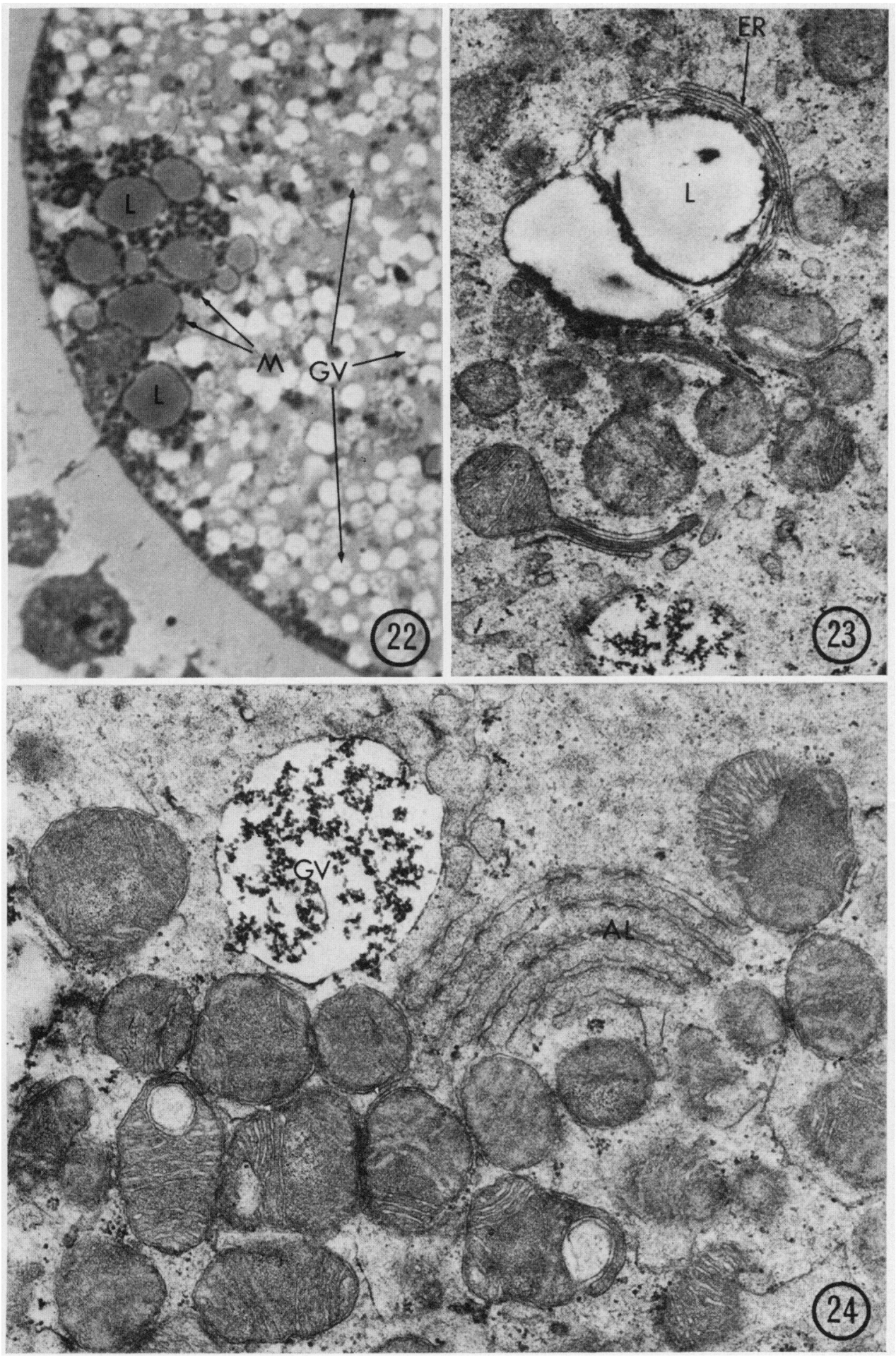


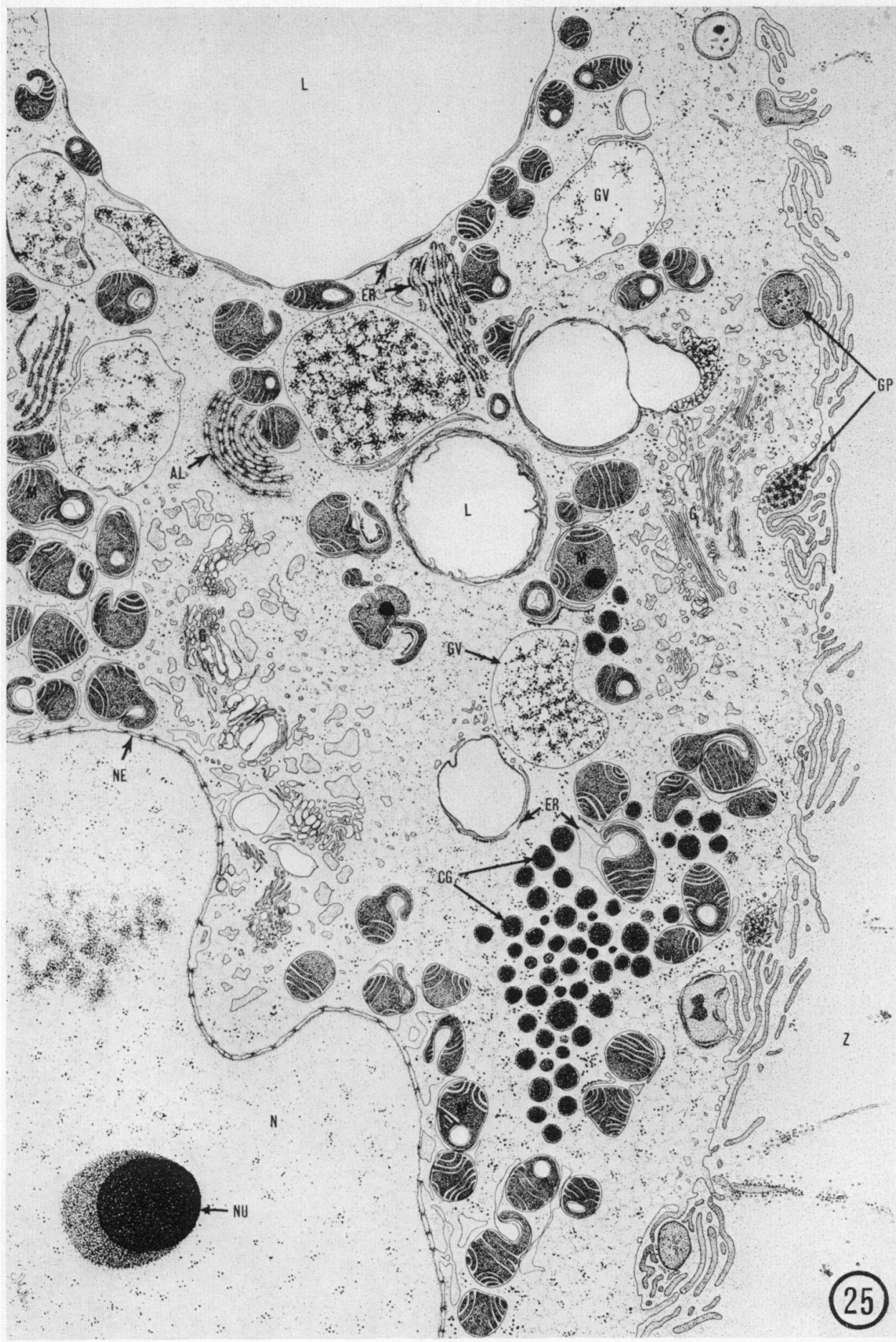


resolved. The vesicles were always found to be uniformly distributed throughout the ooplasm.

Plate 8, Fig. 25 is a graphic illustration summarizing the observations in this study. The illustration demonstrates the interrelationship between organelles and inclusions observed in the bovine oocyte aspirated from the mature Graafian follicle during behavioural oestrus. The illustration is of a Type I oocyte. While distribution of organelles and inclusions varied, the ultrastructural relationships among them were not altered during the final maturation of the oocyte.

\section{DISGUSSION}

The peripheral distribution of organelles in Type-I oocytes was comparable to that reported in oocytes from secondary and young tertiary follicles in the rat

\section{EXPLANATION OF PLATES 5 TO 8}

\section{PLATE 5}

Figs. 14 and 16. Both rough and smooth cisternae of the endoplasmic reticulum (ER) are shown in relation to the unique mitochondria. The cisternae are closely apposed to the outer surface of the mitochondrial hood. Cross sections of hooded mitochondria reveal a cavity formed by the hood and portions of the endoplasmic reticulum within the cavity (arrow). $\times 21,840$ and $\times 13,300$.

Figs. 15 and 17. The endoplasmic reticulum is shown entering the mitochondrial hood (arrows) as well as associating with the outer surface of other mitochondria and cytoplasmic inclusions. $\times 35,900$ and $\times 25,600$.

FIG. 18. Continuity between the outer leaflet of the nuclear envelope and the endoplasmic reticulum (ER) was occasionally observed (arrow). $\times 17,500$.

\section{PLATE 6}

Fxc. 19. Distributed throughout the nucleoplasm (N) of Type-I and -II oocytes are dense staining granules, resembling ribosomes. These particles are found singly and in clusters. $\times 12,100$.

FIG. 20. This type of nucleolus (NU) has a well-developed nucleolonema and is characterized by swollen interstices between nucleolonemal strands. $\times 14,500$.

Fig. 21. This nucleolus (NU) is composed of a dense ovoid structure partially encapsulated by a lighter staining cover. $\times 16,300$.

\section{PLATE 7}

Frg. 22. Light micrograph of the peripheral ooplasm of a Type-I oocyte. Lipid droplets (L) are surrounded by aggregates of mitochondria $(M)$ which appear as densely staining granules. Generally distributed throughout the ooplasm are vesicles containing moderately stained granules $(\mathrm{GV}) . \times 3160$.

FrG. 23. Ultrastructurally, smooth endoplasmic reticulum (ER) and a high osmiophilia is often observed at the edge of lipid droplets (L). $\times 17,750$.

Fig. 24. The annulate lamellae (AL) appear as stacks of cisternae that fuse at intervals along the membrane causing this structure to resemble fragments of the nuclear envelope. A granulated vesicle (GV) is also shown and an aggregate of mitochondria. In electron micrographs, the granulated vesicles contain densely staining granules surrounded by a single membrane. $\times 17,750$.

\section{PLATE 8}

FIG. 25. This is a graphic illustration summarizing the structure and interrelationship of organelles and inclusions observed in the bovine oocyte from the mature Graafian follicle.

Abbreviations of organelles and inclusions: $\mathrm{AL}=$ annulate lamellae, $\mathrm{CG}=$ cortical granules, $\mathrm{ER}=$ endoplasmic reticulum, $\mathrm{G}=\mathrm{Golgi}$ dictyosomes, $\mathrm{GV}=$ granulated vesicle, $\mathrm{L}=$ lipid droplet, $\mathrm{M}=$ mitochondria, $\mathrm{N}=$ nucleus, $\mathrm{NE}=$ nuclear envelope, $\mathrm{NU}=$ nucleolus, $\mathrm{GP}=$ granulosa-cell processes, $\mathrm{Z}=$ zona pellucida. 
(Sotelo \& Porter,1959; Odor, 1960). It seems reasonable that this distribution may be related to the intimate association between the oocyte and granulosa cells. Supporting this concept were the numerous processes in the peripheral ooplasm and on the surface of the oolemma in Type-I oocytes. Most likely, the granulosa-cell processes undulate or coil on the surface of the oocyte or in the peripheral ooplasm. This interpretation is based on the frequent appearance of cross sections of granulosa-cell processes at the surface of oocytes and within the peripheral ooplasm and the relatively infrequent observations of sagittal sections. Such an arrangement would foster maximum surface contact between the granulosa cells and the oocytes. In Type-I oocytes, evidence that the intimate relationship between the granulosa cell and oocyte was deteriorating could be found. The deterioration of the processes was apparent at the interface of the zona pellucida and oolemma but not where the processes were surrounded by ooplasm nor where they traversed the zona. Progressive deterioration and withdrawal of granulosa-cell processes observed in Type-II, -III and -IV oocytes indicate that the strong relationship between the oocyte and granulosa cell is terminated during behavioural oestrus in the bovine.

The shift to a homogeneous distribution of mitochondria and lipid droplets in Type-II compared to Type-I oocytes was accompanied by the appearance of the perivitelline space, which, in turn, was related to further deterioration and withdrawal of granulosa-cell processes. This shift in organelle and inclusion distribution reflects a possible change in the activity of the oocyte. It is very likely that the breakdown in relationship between the oocyte and the surrounding granulosa cells results in a shift of activity within the oocyte from an extracellular dependence to an intracellular one.

In oocytes in which the nuclear envelope was still intact (Types I and II), the nuclei were eccentrically located. The chromosomes in these prophase nuclei appeared as dispersed aggregates of densely staining granules and resembled the chromatin material observed in prophase nuclei of human oocytes from primary follicles (Hertig \& Adams, 1967).

Continuity between the outer leaflet of the nuclear envelope and endoplasmic reticulum was occasionally observed. These observations were very similar to those of Hertig \& Adams (1967) in their study of human oocytes from primary follicles. Similar observations in somatic cells have resulted in the speculation by Fawcett (1966a) that this continuity may be important in facilitating exchange of material between the nucleoplasm and the cytoplasm. Such a relationship has also been offered as indirect evidence for the generation of new endoplasmic reticulum from the nuclear envelope (Gay, 1956).

The two nucleoli having a well-developed nucleolonema observed in the present study resembled reports of nucleoli in the prophase nucleus of other mammalian oocytes (Anderson \& Beams, 1960; Blanchette, 1961; Hertig \& Adams, 1967). The dense ovoid nucleoli, which were much more common within oocytes in this study, have been observed in pronuclei of penetrated ova from rats (Sotelo \& Porter, 1959) and rabbits (Hadek, 1965; Zamboni \& Mastroianni, 1966), but have not been previously reported for unfertilized mammalian oocytes. In amphibian oocytes, nucleoli of this type were shown to produce precursors for r-RNA molecules (Miller \& Beatty, 1969). These 
workers described the nucleolus as a densely staining core surrounded by lighter staining granular material. The dense core was primarily DNA with the surrounding granular cortex being composed of DNA and RNA. The close resemblance of the dense nucleolus of the bovine oocyte to those described in amphibian oocytes suggests that a similar function may be ascribed to the bovine nucleolus. The production of ribosomal RNA precursor would be very important for the rapid development of the embryo following fertilization.

In contrast to Type-I and -II oocytes, the condensed chromatin and absence of a nuclear envelope in Type-III oocytes undoubtedly represented a more advanced stage of maturation in preparation for the first reduction division. The condensed chromosomes and deteriorated state of the nuclear envelope observed in Type-III oocytes logically precedes formation of the first polar body found in Type-IV oocytes. Hafez \& Ishibashi (1964) have shown that the bovine oocyte forms the first polar body either during late oestrus or before ovulation. The fact that all oocytes in the present study were obtained during behavioural oestrus suggests that the first polar body is shed during the latter portion of this period.

A large percentage of mitochondria had a 'hood-like' appendage which formed an extramitochondrial cavity. These mitochondria had been previously described by Senger \& Saacke (1970b) in a study of bovine oocytes from tertiary follicles. In a few adjacent cells of an otherwise normal rat liver, Stephens \& Bils (1965) found mitochondria with an extramitochondrial cavity which was continuous with the cytoplasmic matrix through a small pore. Serial sections were required to demonstrate this pore. These mitochondria appeared to be similar to the hooded mitochondria observed in the present study though the cavity described by Stephens \& Bils (1965) appeared to be more centrally located. The point of continuity between the mitochondrial cavity and the ooplasm in the bovine oocyte was much more obvious than that described for the liver mitochondria. Micrographs of Senger \& Saacke (1970b) revealed membranes within the mitochondrial cavity of bovine oocytes that they described as vesicles. It is apparent from the present study that these membranes were actually continuous with the cisternae of the endoplasmic reticulum. Ribosomes were occasionally associated with these cisternae. The single cisternae of the endoplasmic reticulum were also very closely associated with the outer surface of mitochondria and cytoplasmic inclusions, particularly lipid droplets. The close association of the cisternae with the outer surface of mitochondria appeared to be very similar to that reported for immature human oocytes (Hertig \& Adams, 1967). The function of the hooded mitochondria, which has been reported for bovine oocytes only, was not apparent. We have observed these same mitochondria in oocytes from tertiary follicles of sheep and goats which suggests an association with ruminants or with metabolic characteristics peculiar to ruminants (W. N. Fleming, unpublished work). This pleomorphic form may also function to increase the surface area of the mitochondria. The close association of the endoplasmic reticulum to the inner surface of the hood also suggests that the hood may provide a specific microenvironment, facilitating the exchange of metabolic intermediates between mitochondria and endoplasmic reticulum. 
The very close association of the endoplasmic reticulum and mitochondria to the surface of lipid droplets suggests that the oocyte may be utilizing lipid stores after the breakdown of the oocyte's association with the granulosa cells. These lipid stores could be important in providing nutrients for the final maturation and fertilization of the oocyte, as well as development of the very early embryo.

Occasionally, the endoplasmic reticulum was observed adjacent to the granulated vesicles. The PAS-positive granules in these vesicles resembled glycogen granules in size and electron density and could also represent a sizeable store of energy. Glycogen has not been detected with the electron microscope in mammalian oocytes, but it has been identified with the light microscope in oocytes from rats (Harter, 1948), hamsters (Oder, 1965) and cows (Moss, Wren \& Sykes 1954). Further work is necessary to establish the chemical nature of the granulated vesicles.

\section{ACKNOWLEDGMENTS}

The authors are grateful to Dr T. L. Bibb for assistance in surgical procedures. The technical assistance of Mrs E. B. Rummel is gratefully acknowledged, and we would also like to thank Mrs J. K. Mullins for preparing the graphic illustration.

\section{REFERENGES}

Anderson, E. \& BeAms, H. W. (1960) Cytological observations on the fine structure of the guinea pig ovary with special reference to the oogonium, primary oocyte and associated follicle cells. 7. Ultrastruct. Res. 3, 432 .

Asdell, S. A. \& Mixner, J. P. (1957) Breeding difficulties in dairy cattle. Physiology. Bull. Cornell Univ. agric. Exp. Stn, 924, 19.

Austin, C. R. (1967) Chromosome deterioration in aging eggs of the rabbit. Nature, Lond. 213, 1018.

BLANGHETtE, J. E. (1961) A study of the fine structure of the rabbit primary oocyte. F. Ultrastruct. Res. 5,349 .

Fawcett, D. W. (1966a) An atlas of fine structure. The cell, p. 140. W. B. Saunders, Philadelphia.

FAwCETt, D. W. (1966b) An atlas of fine structure. The cell, p. 336. W. B. Saunders, Philadelphia.

GAY, H. (1956) Nucleo-cytoplasmic relation in Drosophila. Cold Spring Harb. Symp. quant. Biol. 21, 256.

GURR, E. (1953) A practical manual of medical and biological staining techniques, pp. 139-141. Leonard Hill, London.

HADEK, R. (1965) The structure of the mammalian egg. Int. Rev. Cytol. 18, 29.

HAFEz, E. S. E. \& IsHIBASHI, I. (1964) Maturation division in bovine oocytes following gonadotropin injections. Cytogenetics, 3, 167.

HARTER, B. T. (1948) Glycogen and carbohydrate-protein complexes in the ovary of the white rat during the oestrous cycle. Anat. Rec. 102, 349.

Hertig, A. T. \& Adams, E. C. (1967) Studies on the human oocyte and its follicle: I. Ultrastructural and histochemical observations on the primordial follicle stage. F. Cell Biol. 34, 647.

HunTER, R. H. F. (1967) The effect of delayed insemination on fertilization and early cleavage in the pig. 7. Reprod. Fert. 13, 133.

JEon, K. W. (1965) Simple method for staining and preserving epoxy resin embedded animal tissue sections for light microscopy. Life Sci. 4, 1839.

LucE, S. (1966) Techniques for electron microscopy. A laboratory manual, p. 4. Washington School of Medicine, St. Louis, Missouri.

Marion, G. B., GIER, H. T. \& Ghoudary, J. B. (1968) Micromorphology of the bovine ovarian follicular system. F. Anim. Sci. 27, 451.

MilleR, O. J., JR \& BEATTY, B. R. (1969) Visualization of nucleolar genes. Science, N.Y. 23, 955.

Mollenhauer, H. H. \& MorkE, D. J. (1966) Golgi apparatus and plant secretion. A. Rev. Pl. Physiol. $17,27$. 
Moss, S., Wren, T. R. \& SyKes, J. F. (1954) Some histological and histochemical observations of the bovine ovary during the estrous cycle. Anat. Rec. 120, 409.

Odor, D. L. (1960) Electron microscopic studies on ovarian oocytes and unfertilized tubal ova in the rat. F. biophys. biochem. Cytol. 7, 567.

Odor, D. L. (1965) The ultrastructure of unilaminar follicles of the hamster ovary. Am. F. Anat. 116, 493.

Senger, P. L. \& SAAGKe, R. G. (1970a) A method for preparing single mammalian ova for cytological studies using light and electron microscopy. 7. Reprod. Fert. $21,177$.

Senger, P. L. \& SAAcke, R. G. (1970b) Unique mitochrondria in the bovine oocyte. J. Cell Biol. 46, 405.

Sotelo, J. R. \& Porter, K. R. (1959) An electron microscope study of the rat ovum. 7. biophys. biochem. Cytol. 5, 327.

Stephens, R. J. \& Birs, R. F. (1965) An atypical mitochondrial form in normal rat liver. F. Cell Biol. 24, 500 .

TANABE, T. Y. \& CAsidA, L. E. (1949) The nature of reproduction failures in cows of low fertility. 7. Dairy Sci. 32, 237.

Venable, J. H. \& Coggeshatr, R. (1965) A simplified lead citrate stain for use in electron microscopy. 7. Cell Biol. 25, 407.

Zamboni, L. \& MAstrolanni, L., JR (1966) Electron microscopic studies on rabbit ova: II. The penetrated tubal ovum. F. Ultrastruct. Res. 14, 118. 